\title{
RENAL FUNCTION, ELECTROLYTE EXCRETION AND BODY \\ FLUIDS IN PATIENTS WITH CHRONIC RENAL IN- SUFFICIENCY BEFORE AND AFTER SODIUM DEPRIVATION 1,2
}

\author{
By JAMES F. NICKEL,8, * PRESTON B. LOWRANCE,5, 6 EDGAR LEIFER, AND \\ STANLEY E. BRADLEY

\begin{abstract}
(From the Department of Medicine, Columbia University College of Physicians and Surgeons and the Presbyterian Hospital, New York, N. Y.)
\end{abstract}

(Submitted for publication September 12, 1952; accepted October 31, 1952)

L'azotémie par manque de sel is a descriptive phrase coined by Blum, Graber, and van Caulaert $(1,2)$ to describe the azotemia and hypochloremia which developed in certain diabetic patients maintained on low salt diets immediately after recovery from coma. When sodium chloride was administered to these patients the plasma chloride concentration increased and nitrogen retention diminished or cleared. Pre-existing renal disease, anuria, or acidosis per se were not contributory factors. Blum and his associates (2) concluded that the development of azotemia was a compensatory response to decreased molecular concentration and osmotic activity of the blood. This view has not been widely accepted because it is known (3) that all membranes in the body are freely permeable to urea. Subsequent work with man (4) and other animals (5) has revealed that severe salt depletion is usually associated with loss and redistribution of body water that may lead to circulatory collapse and renal dysfunction.

Marked sodium depletion is necessary to induce renal vasoconstriction and diminished glomerular filtration sufficient to cause nitrogen retention in normal human subjects and it is usually associated with obvious dehydration, weight loss, muscular weakness, apathy, and anorexia (4). In patients with renal insufficiency secondary to renal disease much less severe sodium depletion suffices to pre-

1 These studies have been made possible through the aid of a grant from the New York Heart Association.

2 Preliminary report of some of these data has been published (37).

${ }^{8}$ Fellow of the New York Heart Association (1950-51) and Life Insurance Medical Research Fund Fellow (195152).

4 Present address: Barnes Hospital, St. Louis, Missouri.

- John and Mary Markle Scholar in Medicine.

- Present address: University of Virginia School of Medicine, Charlottesville, Virginia. cipitate or to intensify nitrogen retention (6). Since renal disease often appears to predispose to easy loss of body water, dehydration may also be an important factor in this situation.

Wiggins, Manry, Lyons, and Pitts (7) have found that moderate salt depletion induces almost complete cessation of sodium excretion in normal man, chiefly as a result of increased tubular reabsorption. In the uremic patient it is possible that this mechanism is deranged and that changes in filtration are necessary to prevent undue loss of sodium in the urine. An investigation was therefore undertaken to define the relative importance of changes in the volume of body fluids, in the distribution of sodium and potassium in the body, and in renal function in the production of increased azotemia during moderate sodium deprivation in patients with chronic renal insuffciency

\section{METHODS}

Two subjects of these studies were normal men clinically free from cardiovascular or renal disease; eight subjects suffered from chronic renal insufficiency with nitrogen retention as a result of well-established chronic diffuse glomerulonephritis (four), malignant arteriolar nephrosclerosis (two), chronic pyelonephritis (one) and polycystic renal disease (one). All were free of edema or other evidences of heart failure. Arterial hypertension had developed in all and anemia in seven.

Deprivation without depletion of sodium was effected by placing five uremic subjects (G. B., M. B., C. P., J. F., and T. C.) on a low salt diet (18 to $25 \mathrm{mEq}$. of sodium per day and $1 \mathrm{Gm}$. protein per $\mathrm{Kg}$. body weight) and sodium-exchanging resin " $60 \mathrm{gm}$. per day in divided doses) for a five day period. With one exception all were maintained on a regular hospital diet containing 70 to $105 \mathrm{mEq}$. of sodium per day for at least one week prior to sodium deprivation. In the exceptional instance (T. C.)

7 We are indebted to Dr. K. G. Kohlstaedt and the Eli Lilly Co., Indianapolis, Indiana, for a generous supply of carboxylic acid exchange resins (Carbo-Resin). 
a low sodium diet (23.6 mEq. sodium per day) had been provided for three days. The low salt diet was discontinued and supplemented by salt tablets $(85 \mathrm{mEq}$. sodium per day) for five days following termination of resin intake in three patients (C. P., J. F., and T. C.) in order to examine the effect of salt repletion. In one uremic subject ( $T$. C.) accurate measurements of the sodium, potassium and water balance were made while in residence on the Metabolism Service of the Presbyterian Hospital. One of the two normal subjects (A. B.) was also studied in the same manner during a five day period of more rigorous sodium withdrawal (18.5 mEq. sodium per day and $120 \mathrm{gm}$. of cation-exchange resin daily in divided doses) followed by sodium repletion as described above.

Measurements of inulin, $p$-aminohippurate (PAH), sodium, potassium, chloride and phosphate clearances and determination of the maximal rate of tubular PAH secretion $\left(\mathrm{Tm}_{\mathrm{pah}}\right)(8,9)$ were made just before and at the close of the periods of sodium deprivation and repletion. In addition, plasma carbon dioxide content (10), plasma calcium concentration (11), and blood urea nitrogen (12) were measured at these times. The urinary outputs of sodium and potassium were measured daily in all. Fecal losses of sodium and potassium were also determined in the two subjects on "balance study" on the Metabolism Service. Sodium and potassium concentrations in plasma and urine were determined with an internal standard flame photometer (13), chloride by the method of Schales and Schales (14), inorganic phosphate by the method of Fiske and Subbarow (15) and $\mathrm{pH}$ (Cambridge glass electrode) in urine samples collected under oil.

The day preceding or following clearance study measurements of the volume of distribution of antipyrine ${ }^{\circ}(16)$, inulin (17), and T-1824 (18) were made in five patients with renal insufficiency. PAH was found to interfere markedly with antipyrine determinations and PAH clearances could not be measured simultaneously. Since inulin excretion was greatly reduced in all these individuals the difference between the amount of inulin excreted during equilibrium and the amount injected was large enough to measure accurately. The amount trapped in the renal "dead space" was relatively small and the method of Gaudino, Schwartz, and Levitt (17) could be safely modified as follows : 1) exactly five grams of inulin in five per cent dextrose were administered by intravenous infusion over a two-hour period; 2) urine was collected simultaneously by indwelling catheter during this period and for the three hours thereafter allowed for equilibration; 3 ) the plasma inulin concentration (mg. per ml.) determined at this time (five hours after beginning the inulin infusion) was divided into the total amount remaining in the body (the amount infused less the amount excreted in the urine during the five hour period) to obtain values for

8 We wish to thank Dr. W. Boger and the Medical Research Division of Sharp and Dohme, Inc., Philadelphia, Penna., for a generous supply of sodium $p$-aminohippurate.

- Supplied through the courtesy of Dr. K. G. Kohlstaedt and the Eli Lilly Co., Indianapolis, Indiana. the volume of distribution of inulin. In every instance, the plasma inulin concentration at "equilibrium" was higher than $20 \mathrm{mg}$. per cent. The "intracellular fluid volume" was estimated as the difference between the inulin and antipyrine "spaces." The hematocrit was determined using venous blood obtained without stasis.

"Total exchangeable" sodium and potassium were determined $(19,20)$ in three uremic subjects by isotope dilution ( $\mathrm{Na}^{2}, 1.2 \mu \mathrm{c}$. per $\mathrm{Kg}$. body weight; $\mathrm{K}^{\circ}, 1.5 \mu \mathrm{c}$. per $\mathrm{Kg}$. body weight). The isotope was injected intravenously, urine carefully collected over a 24 hour period and the "exchangeable" mass computed from the plasma specific activity at the end of this period and the amount of isotope remaining in the body, allowing for decay and elimination in the urine. In three subjects the volume of distribution of $\mathrm{Na}^{24}$ four hours after injection (21) was determined for comparison with simultaneously determined inulin "space." The distribution of "total exchangeable" sodium and potassium between "cells" (and bone) and extracellular fluid was calculated on the basis of simultaneously determined inulin and antipyrine "spaces."

The body weight was measured daily and the blood pressure determined sphygmomanometrically twice daily throughout each study.

\section{RESULTS}

Electrolyte and water excretion during renal insufficiency. The data presented in Tables I to III, obtained in studies of eight patients with chronic renal insufficiency, show that sodium, potassium, chloride and water excretion were usually increased above normal, relative to glomerular filtration. The greater loss of filtered electrolyte in the urine (electrolyte-inulin clearance ratio, $\mathrm{C}_{\mathrm{e}} / \mathrm{C}_{\mathrm{in}}$, or the percentage of filtered electrolyte excreted) is indicative of diminished tubular reabsorption that serves to maintain electrolyte balance. Normally, little more than 25 per cent of the water, sodium and chloride in the glomerular filtrate appears in the urine even under heavy loads for excretion (22), whereas as much. as 63 per cent of water, 18 per cent of sodium, and 17 per cent of chloride in the filtrate (Tables I and II) were excreted by patients in this series at rest. Potassium excretion fell into a special category because it actually exceeded the amount filtered in five of the eight subjects (Table I), in contrast to the normal where more than 20 per cent seldom appears in the urine (23) except under conditions of loading. This phenomenon has also been described by Keith (24), Earle (25), Platt (26) and their colleagues who interpret it as evidence for active tubular secretion of potassium. These 
defects in renal electrolyte excretion were not associated with any serious disturbance in plasma electrolyte composition in these selected patients (Table III).

The maximal tubular PAH secretion was reduced to approximately the same extent as filtration, the inulin clearance- $\mathrm{Tm}_{\mathrm{pah}}$ ratio (which normally averages $1.5 \mathrm{ml}$. per $\mathrm{mg}$.) amounting to $1.76,1.50,0.70,1.18$, and $1.35 \mathrm{ml}$. filtrate per $\mathrm{mg}$. of secreted PAH (Table III) in the five subjects in whom the measurement was made. A study of electrolyte excretion in three of these subjects during $\mathrm{PAH}$ revealed (Table II-control studies) little or no changes in $\mathrm{pH}$, phosphate clearance $\left(\mathrm{C}_{\mathrm{p}} / \mathrm{C}_{\text {in }}\right)$ and urine flow $\left(\mathrm{V} / \mathrm{C}_{\text {in }}\right)$ relative to the inulin clearance. In one normal subject (A. C.control, Table II) studied under the same conditions, $\mathrm{pH}$ fell sharply and both $\mathrm{C}_{\mathrm{p}} / \mathrm{C}_{\mathrm{in}}$ and $\mathrm{V} / \mathrm{C}_{\mathrm{in}}$ increased, as noted previously by West and Rapoport (27). The percentage of filtered sodium, potassium and chloride excreted in the urine $\left(\mathrm{C}_{\mathrm{na}} / \mathrm{C}_{\mathrm{in}}, \mathrm{C}_{\mathrm{k}} / \mathrm{C}_{\mathrm{in}}\right.$, and $\left.\mathrm{C}_{\mathrm{cl}} / \mathrm{C}_{\mathrm{in}}\right)$ increased during $\mathrm{PAH}$ loading in both the normal and uremic subjects (with the exception of M. B., Table II-in whom $\mathrm{C}_{\mathrm{k}} / \mathrm{C}_{\mathrm{in}}$ fell).

Effect of sodium deprivation on electrolyte ex-

TABLE I

Proportion of filtered water and electrolytes excreted in the urine of patients with chronic renal insufficiency

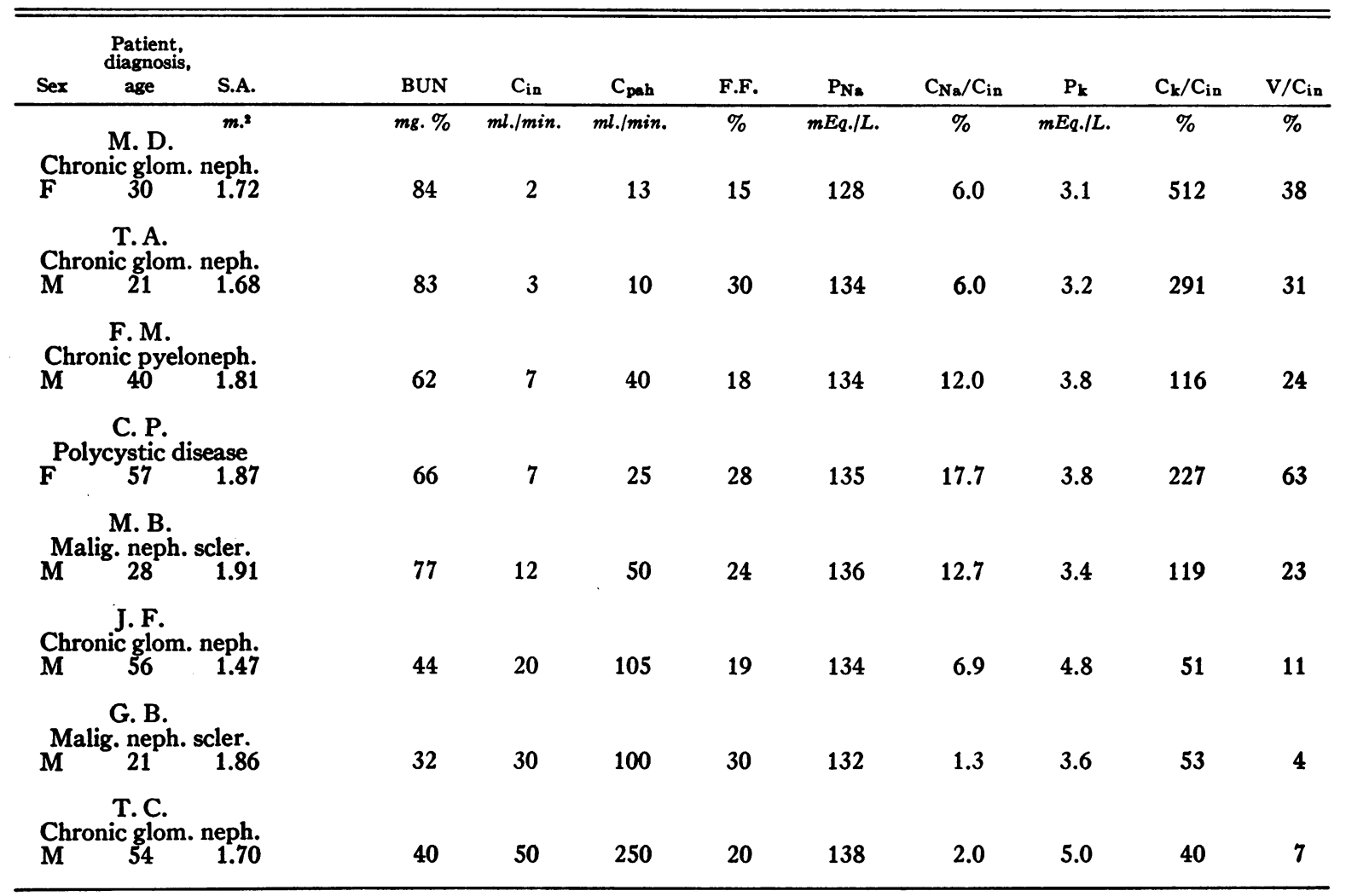

All values for renal clearances and urinary sodium and potassium excretion are averages of three successive determinations.

Abbreviations are as follows:

S.A. = Surface Area $\left(\mathbf{M}^{2}\right)$.

$\mathrm{C}_{\text {in }}=$ Inulin clearance.

$\mathrm{C}_{\mathrm{pah}}=$ Sodium $p$-aminohippurate clearance

$\mathrm{FF}=$ Filtration fraction $\left(\mathrm{C}_{\text {in }} / \mathrm{C}_{\mathrm{pah}}\right)$.

$\mathrm{P}_{\mathrm{N}_{\mathrm{a}}}=$ Plasma sodium concentration.

$\mathrm{C}_{\mathrm{Na}} / \mathrm{C}_{\mathrm{in}}=$ Sodium-inulin clearance ratio (percentage of filtered sodium excreted).

$P_{k}=$ Plasma potassium concentration.

$C_{k} / C_{i n}=$ Potassium-inulin clearance ratio (percentage of filtered potassium excreted).

$\mathrm{V} / \mathrm{C}_{\mathrm{in}}=$ Urine flow-inulin clearance ratio (percentage of filtered water excreted; no correction for free water in the plasma has been made, since the error introduced is negligible). 
cretion. Sodium deprivation by low salt diet and sodium-exchange resin resulted in a sharp decrease in sodium excretion $\left(\mathrm{U}_{\mathrm{Na}} \mathrm{V}\right.$-mg. per min., Table III) to 2.8 per cent of control in one normal subject (A. C.-control, Table III, preliminary control figures were not obtained in A. B.) and to 36.0, $72.5,20.2$, and 32.2 per cent of control in four of five uremic patients (Table III). There was no significant change in sodium output in C. P., a woman with long-standing renal insufficiency due to polycystic disease. Potassium output fell from $53.1 \mu \mathrm{Eq}$. per min. to $10.4 \mu \mathrm{Eq}$. per min. in the normal subject (A. C.-Table III) but showed no consistent change in direction or magnitude among the uremics, rising in C. P. from 58.6 to $98.3 \mu \mathrm{Eq}$. per min. and in M. B. from 47.2 to
$58.2 \mu$ Eq. per min., falling in J. F. from 47.7 to $33.4 \mu \mathrm{Eq}$. per min., and remaining relatively unchanged in the other patients (G. B. and T. C.).

The values for electrolyte excretion given in Tables I to III are those measured during determination of clearances and are not to be construed as averages or representative of the output during the course of each day. The methods of measurement, diurnal variation (28), and other factors might be expected to affect these figures, but since they were obtained under standard conditions it seems reasonable to attribute any difference between values before and after deprivation to the effect of deprivation alone. The average minute output of sodium during each day of deprivation was probably much less than the values

TABLE II

The effect of intravenous $P A H$ loading on urine $p H$ and urinary electrolyte excretion

\begin{tabular}{|c|c|c|c|c|c|c|c|c|c|}
\hline Patient & & Regimen & $\mathrm{C}_{\mathrm{in}}$ & $\mathrm{pH}$ & $\mathrm{C}_{\mathrm{Na}} / \mathrm{C}_{\mathrm{in}}$ & $\mathrm{C}_{\mathrm{k}} / \mathrm{C}_{\mathrm{in}}$ & $\mathrm{C}_{\mathrm{C} 1 / \mathrm{C}_{\mathrm{in}}}$ & $C_{p} / C_{i n}$ & $\mathrm{~V} / \mathrm{C}_{\mathrm{in}}$ \\
\hline & & & ml./min. & units & & & $\%$ & & \\
\hline \multicolumn{10}{|c|}{ Normal Renal Status } \\
\hline A. C. & $\begin{array}{l}\text { Control } \\
\mathrm{Na} \text { depriv. }\end{array}$ & $\begin{array}{l}\text { Before } \\
\text { PAH load } \\
\text { Before } \\
\text { PAH load }\end{array}$ & $\begin{array}{r}105 \\
70 \\
105 \\
95\end{array}$ & $\begin{array}{l}6.9 \\
5.4 \\
6.3 \\
4.7\end{array}$ & $\begin{array}{l}1.45 \\
4.16 \\
0.04 \\
1.00\end{array}$ & $\begin{array}{r}14.80 \\
17.90 \\
3.20 \\
6.90\end{array}$ & $\begin{array}{l}1.25 \\
1.57 \\
0.25 \\
0.32\end{array}$ & $\begin{array}{r}8.55 \\
17.55 \\
14.03 \\
19.43\end{array}$ & $\begin{array}{l}1.63 \\
3.20 \\
5.00 \\
5.69\end{array}$ \\
\hline A. B. & $\begin{array}{l}\text { Na depriv. } \\
\text { Repletion }\end{array}$ & $\begin{array}{l}\text { Before } \\
\text { PAH load } \\
\text { Before } \\
\text { PAH load }\end{array}$ & $\begin{array}{r}120 \\
80 \\
125 \\
120\end{array}$ & $\begin{array}{l}6.5 \\
4.7 \\
5.8 \\
5.0\end{array}$ & $\begin{array}{l}0.01 \\
0.39 \\
0.14 \\
1.43\end{array}$ & $\begin{array}{r}0.93 \\
10.71 \\
7.55 \\
11.47\end{array}$ & $\begin{array}{l}0.55 \\
0.72 \\
0.51 \\
0.87\end{array}$ & $\begin{array}{r}7.10 \\
19.14 \\
3.55 \\
10.03\end{array}$ & $\begin{array}{l}1.38 \\
3.73 \\
0.53 \\
1.23\end{array}$ \\
\hline \multicolumn{10}{|c|}{ Chronic Renal Insufficiency } \\
\hline M. B. & $\begin{array}{l}\text { Control } \\
\mathrm{Na} \text { depriv. }\end{array}$ & $\begin{array}{l}\text { Before } \\
\text { PAH load } \\
\text { Before } \\
\text { PAH load }\end{array}$ & $\begin{array}{r}12 \\
14 \\
7 \\
6\end{array}$ & $\begin{array}{l}6.3 \\
6.3 \\
6.2 \\
6.2\end{array}$ & $\begin{array}{l}12.70 \\
18.20 \\
17.00 \\
25.30\end{array}$ & $\begin{array}{l}118.90 \\
102.30 \\
249.00 \\
259.70\end{array}$ & $\begin{array}{l}16.90 \\
21.10 \\
23.70 \\
31.30\end{array}$ & $\begin{array}{l}9.71 \\
9.79 \\
5.56 \\
5.14\end{array}$ & $\begin{array}{l}22.50 \\
28.10 \\
36.60 \\
40.90\end{array}$ \\
\hline J. F. & $\begin{array}{l}\text { Control } \\
\mathrm{Na} \text { depriv. } \\
\text { Repletion }\end{array}$ & $\begin{array}{l}\text { Before } \\
\text { PAH load } \\
\text { Before } \\
\text { PAH load } \\
\text { Before } \\
\text { PAH load }\end{array}$ & $\begin{array}{l}20 \\
19 \\
12 \\
12 \\
26 \\
23\end{array}$ & $\begin{array}{l}5.7 \\
5.7 \\
4.8 \\
4.7 \\
5.7 \\
5.7\end{array}$ & $\begin{array}{r}6.90 \\
21.20 \\
2.30 \\
6.70 \\
7.90 \\
14.70\end{array}$ & $\begin{array}{l}50.80 \\
62.30 \\
61.60 \\
80.30 \\
51.20 \\
56.30\end{array}$ & $\begin{array}{r}9.10 \\
12.00 \\
3.70 \\
4.70 \\
9.70 \\
14.50\end{array}$ & $\begin{array}{l}11.50 \\
13.80 \\
10.60 \\
10.30 \\
15.30 \\
14.20\end{array}$ & $\begin{array}{l}10.70 \\
12.20 \\
23.00 \\
31.70 \\
13.50 \\
22.60\end{array}$ \\
\hline T. C. & $\begin{array}{l}\text { Control } \\
\text { Na depriv. } \\
\text { Repletion }\end{array}$ & $\begin{array}{l}\text { Before } \\
\text { PAH load } \\
\text { Before } \\
\text { PAH load } \\
\text { Before } \\
\text { PAH load }\end{array}$ & $\begin{array}{l}50 \\
40 \\
30 \\
30 \\
45 \\
45\end{array}$ & $\begin{array}{l}5.2 \\
5.3 \\
4.4 \\
4.6 \\
4.9 \\
5.0\end{array}$ & $\begin{array}{r}1.95 \\
7.85 \\
0.85 \\
5.16 \\
4.05 \\
12.26\end{array}$ & $\begin{array}{r}39.50 \\
54.80 \\
67.00 \\
102.50 \\
28.10 \\
34.90\end{array}$ & $\begin{array}{r}3.28 \\
6.26 \\
1.42 \\
2.72 \\
2.80 \\
11.36\end{array}$ & $\begin{array}{l}15.30 \\
18.91 \\
21.53 \\
19.27 \\
18.61 \\
21.29\end{array}$ & $\begin{array}{r}6.65 \\
11.72 \\
7.80 \\
10.07 \\
4.22 \\
10.86\end{array}$ \\
\hline
\end{tabular}

All values are averages of three determinations.

Abbreviations are as follows; otherwise as in Table I:

Regimen: Control, on diet containing 70 to $105 \mathrm{mEq}$. sodium daily, at least $1 \mathrm{gm}$. protein per $\mathrm{Kg}$. daily, and adequate caloric provision;

$\mathrm{Na}$ depriv., sodium intake reduced to 18 to $25 \mathrm{mEq}$. daily, and cation exchange resin (Carbo-resin) $60 \mathrm{gm}$. daily in divided doses;

Repletion, control diet and supplement of $85 \mathrm{mEq}$. sodium daily. Each study at termination of a

$\mathrm{C}_{\mathrm{or}} / \mathrm{C}_{\mathrm{in}}$ : Chloride-inulin clearance ratio (percentage of filtered chloride excreted).

$\mathrm{C}_{\mathrm{p}} / \mathrm{C}_{\mathrm{in}}$ : Phosphate-inulin clearance ratio (percentage of filtered phosphate excreted). 
TABLE III

The effect of sodium deprivation on body weight, renal function, urinary sodium and potassium excretion, and on plasma electrolytes

\begin{tabular}{|c|c|c|c|c|c|c|c|c|c|c|c|c|c|c|c|c|}
\hline Sex & $\begin{array}{c}\begin{array}{c}\text { Patient } \\
\text { Diagnosis } \\
\text { Age }\end{array} \text { S.A. } \\
\text { P. }\end{array}$ & Regimen* & $\begin{array}{l}\text { Body } \\
\text { wt. }\end{array}$ & $\mathrm{C}_{\text {in }}$ & $\mathrm{C}_{\mathrm{pah}}$ & F.F. & $T m_{p a h}$ & $\mathrm{UNaV}_{\mathrm{Na}}$ & $\mathbf{U}_{\mathbf{k}} \mathbf{V}$ & $\mathrm{Na}$ & $\mathbf{K}$ & $\mathrm{CO}_{2}$ & $\begin{array}{l}\text { Plasma } \\
\mathrm{Cl}\end{array}$ & $\mathrm{Ca}$ & $\mathbf{P}$ & BUN \\
\hline & $m . .^{2}$ & & $K_{\boldsymbol{g}}$ & $\begin{array}{l}\operatorname{ml.l} \\
\min .\end{array}$ & min. & $\%$ & $\begin{array}{l}\text { mg.l } \\
\text { min. }\end{array}$ & $\begin{array}{l}\mu E q . / \\
\min .\end{array}$ & $\begin{array}{l}\mu E q .1 \\
\min .\end{array}$ & \multicolumn{3}{|c|}{$m E q . / L$} & \multicolumn{4}{|c|}{$m g . \%$} \\
\hline \multicolumn{17}{|c|}{ Normal Renal Status } \\
\hline$\underset{\mathbf{F}}{\mathrm{Rh}}$ & $\underset{37}{2} 1.49$ & $\begin{array}{l}\text { Control } \\
\text { Na depriv. }\end{array}$ & $\begin{array}{l}43.6 \\
42.8\end{array}$ & $\begin{array}{l}105 \\
105\end{array}$ & $\begin{array}{l}755 \\
550\end{array}$ & $\begin{array}{l}14 \\
19\end{array}$ & $\begin{array}{l}65 \\
35\end{array}$ & $\begin{array}{r}203.6 \\
5.8\end{array}$ & $\begin{array}{l}53.1 \\
10.4\end{array}$ & $\begin{array}{l}137 \\
134\end{array}$ & $\begin{array}{l}3.5 \\
3.1\end{array}$ & $\begin{array}{l}24.2 \\
22.9\end{array}$ & $\begin{array}{l}99.7 \\
99.4\end{array}$ & $\begin{array}{r}9.4 \\
10.0\end{array}$ & $\begin{array}{l}4.1 \\
4.2\end{array}$ & $\begin{array}{l}10 \\
11\end{array}$ \\
\hline $\begin{array}{l}\text { Pul } \\
\text { M }\end{array}$ & $\begin{array}{l}\text { A. B. } \\
\text { n. emphysema } \\
45 \\
1.70\end{array}$ & $\begin{array}{l}\text { Na depriv. } \\
\text { Repletion }\end{array}$ & $\begin{array}{l}64.7 \\
66.2\end{array}$ & $\begin{array}{l}120 \\
125\end{array}$ & $\begin{array}{l}505 \\
575\end{array}$ & $\begin{array}{l}24 \\
23\end{array}$ & $\begin{array}{l}40 \\
50\end{array}$ & $\begin{array}{r}1.2 \\
27.3\end{array}$ & $\begin{array}{r}3.7 \\
48.9\end{array}$ & $\begin{array}{l}137 \\
140\end{array}$ & $\begin{array}{l}4.2 \\
5.0\end{array}$ & $\begin{array}{l}28.8 \\
26.4\end{array}$ & $\begin{array}{r}96.8 \\
103.0\end{array}$ & $\begin{array}{l}9.7 \\
9.4\end{array}$ & $\begin{array}{l}3.1 \\
3.3\end{array}$ & $\begin{array}{l}15 \\
13\end{array}$ \\
\hline \multicolumn{17}{|c|}{ Chronic Renal Insufficiency } \\
\hline$\underset{\mathbf{M}}{\mathbf{M}}$ & ${ }_{21} \quad 1.86$ & $\begin{array}{l}\text { Control } \\
\text { Na depriv. }\end{array}$ & $\begin{array}{l}62.6 \\
62.5\end{array}$ & $\begin{array}{l}30 \\
15\end{array}$ & $\begin{array}{r}100 \\
60\end{array}$ & $\begin{array}{l}30 \\
25\end{array}$ & $\begin{array}{r}17 \\
8\end{array}$ & $\begin{array}{l}48.7 \\
17.5\end{array}$ & $\begin{array}{l}\mathbf{5 3 . 0} \\
51.3\end{array}$ & $\begin{array}{l}132 \\
132\end{array}$ & $\begin{array}{l}3.6 \\
3.0\end{array}$ & $\begin{array}{l}29.3 \\
28.0\end{array}$ & $\begin{array}{l}94.8 \\
93.0\end{array}$ & $\begin{array}{l}9.5 \\
9.1\end{array}$ & $\begin{array}{l}5.1 \\
5.4\end{array}$ & $\begin{array}{l}32 \\
30\end{array}$ \\
\hline$\underset{\mathbf{M}}{\mathbf{M}}$ & $\begin{array}{l}\text { M. B. } \\
\underset{28}{\text { lig. neph. scl. }} 1.91\end{array}$ & $\begin{array}{l}\text { Control } \\
\text { Na depriv. }\end{array}$ & $\begin{array}{l}68.5 \\
67.5\end{array}$ & $\begin{array}{r}12 \\
7\end{array}$ & $\begin{array}{l}50 \\
25\end{array}$ & $\begin{array}{l}24 \\
28\end{array}$ & $\begin{array}{l}8 \\
4\end{array}$ & $\begin{array}{l}205.3 \\
149.3\end{array}$ & $\begin{array}{l}47.2 \\
58.2\end{array}$ & $\begin{array}{l}136 \\
132\end{array}$ & $\begin{array}{l}3.4 \\
3.4\end{array}$ & $\begin{array}{l}22.6 \\
23.0\end{array}$ & $\begin{array}{l}96.4 \\
91.2\end{array}$ & $\begin{array}{l}9.1 \\
8.7\end{array}$ & $\begin{array}{r}9.4 \\
10.4\end{array}$ & $\begin{array}{r}77 \\
102\end{array}$ \\
\hline Po & 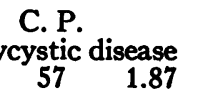 & $\begin{array}{l}\text { Control } \\
\text { Na depriv. } \\
\text { Repletion }\end{array}$ & $\begin{array}{l}82.0 \\
81.2 \\
84.0\end{array}$ & $\begin{array}{r}7 \\
8 \\
12\end{array}$ & $\begin{array}{l}25 \\
15 \\
25\end{array}$ & $\begin{array}{l}28 \\
48 \\
45\end{array}$ & $\begin{array}{r}10 \\
4 \\
4\end{array}$ & $\begin{array}{l}165.6 \\
162.3 \\
319.5\end{array}$ & $\begin{array}{l}58.6 \\
98.3 \\
91.3\end{array}$ & $\begin{array}{l}135 \\
136 \\
142\end{array}$ & $\begin{array}{l}3.8 \\
3.5 \\
3.4\end{array}$ & $\begin{array}{l}22.0 \\
24.9 \\
23.5\end{array}$ & $\begin{array}{r}103.5 \\
99.8 \\
104.5\end{array}$ & $\begin{array}{l}6.9 \\
7.6 \\
7.3\end{array}$ & $\begin{array}{l}6.9 \\
7.8 \\
7.0\end{array}$ & $\begin{array}{l}66 \\
64 \\
60\end{array}$ \\
\hline$\underset{\mathbf{M}}{\mathrm{Chr}}$ & $\underset{56}{\text { J. F. }} \underset{\text { nic glom. neph. }}{\text { neph }}$ & $\begin{array}{l}\text { Control } \\
\text { Na depriv. } \\
\text { Repletion }\end{array}$ & $\begin{array}{l}50.2 \\
48.6 \\
51.4\end{array}$ & $\begin{array}{l}20 \\
12 \\
26\end{array}$ & $\begin{array}{r}105 \\
95 \\
125\end{array}$ & $\begin{array}{l}19 \\
12 \\
21\end{array}$ & $\begin{array}{l}17 \\
13 \\
13\end{array}$ & $\begin{array}{r}166.9 \\
33.7 \\
277.0\end{array}$ & $\begin{array}{l}47.7 \\
33.4 \\
67.5\end{array}$ & $\begin{array}{l}134 \\
131 \\
136\end{array}$ & $\begin{array}{l}4.8 \\
4.7 \\
5.0\end{array}$ & $\begin{array}{l}21.6 \\
19.1 \\
23.0\end{array}$ & $\begin{array}{l}109.3 \\
107.0 \\
108.2\end{array}$ & $\begin{array}{l}9.1 \\
8.5 \\
9.6\end{array}$ & $\begin{array}{l}4.3 \\
5.5 \\
4.1\end{array}$ & $\begin{array}{l}44 \\
63 \\
37\end{array}$ \\
\hline$\underset{\text { M }}{\mathrm{Chr}}$ & $\begin{array}{l}\text { T. C. } \\
\text { nic glom. neph. } \\
\begin{array}{l}54 \\
1.70\end{array}\end{array}$ & $\begin{array}{l}\text { Control } \\
\text { Na depriv. } \\
\text { Repletion }\end{array}$ & $\begin{array}{l}61.6 \\
60.8 \\
60.9\end{array}$ & $\begin{array}{l}50 \\
30 \\
45\end{array}$ & $\begin{array}{l}250 \\
230 \\
295\end{array}$ & $\begin{array}{l}20 \\
14 \\
16\end{array}$ & $\begin{array}{l}37 \\
39 \\
38\end{array}$ & $\begin{array}{r}129.0 \\
41.6 \\
264.2\end{array}$ & $\begin{array}{r}100.3 \\
98.5 \\
59.7\end{array}$ & $\begin{array}{l}138 \\
137 \\
140\end{array}$ & $\begin{array}{l}5.0 \\
4.1 \\
4.7\end{array}$ & $\begin{array}{l}22.0 \\
19.9 \\
21.9\end{array}$ & $\begin{array}{l}103.0 \\
106.7 \\
104.1\end{array}$ & $\begin{array}{r}9.7 \\
10.1 \\
10.3\end{array}$ & $\begin{array}{l}4.0 \\
5.4 \\
4.4\end{array}$ & $\begin{array}{l}40 \\
65 \\
38\end{array}$ \\
\hline
\end{tabular}

* Regimens as noted under Table II.

All values for renal clearances and urinary sodium and potassium excretion are averages of three determinations.

Abbreviations are as follows; otherwise as in Tables I and II:

$\mathrm{Tm}_{\mathrm{pah}}=$ maximal tubular excretion of PAH.
$\mathrm{U}_{\mathrm{Na}}=$ urinary sodium output.

$\mathrm{U}_{\mathbf{k}} \mathrm{V}=$ urinary potassium output.

for $\mathrm{U}_{\mathrm{na}} \mathrm{V}$ presented in Table III. Thus, careful measurement of sodium balance in one uremic patient (T. C.-Table IV) revealed that urinary sodium excretion during deprivation ranged from 4.5 to $7.4 \mathrm{mEq}$. per day, or from 3.1 to $5.1 \mu \mathrm{Eq}$. per min. on the average, as compared with the value of $41.6 \mu \mathrm{Eq}$. per min. observed during the clearance study.

The resin, in the doses used, assured a loss of sodium in the stool approximately equal, in T. C. (Table IV), to intake, resulting in a slightly negative sodium balance that totalled $28.8 \mathrm{mEq}$. for the five days of deprivation. This small deficit was insufficient to produce any obvious change in plasma sodium concentration (Table III). The resin provided enough potassium to permit a small potassium gain of $15.8 \mathrm{mEq}$. over the five days of administration in T. C. (Table IV) without any effect on plasma potassium concentration. It may be presumed that very small changes in electrolyte balance occurred similarly in the other subjects on the same regimen. This assumption finds support in the absence of much change in the plasma electrolyte pattern (Table III). However, the plasma phosphate concentration ( $P$, Table III) did tend to rise and the blood urea nitrogen increased decidedly in three of the five uremic patients, by 35,43 , and 62 per cent (Table III).

Effect of sodium deprivation on glomerular filtration and tubular function. The increment in nitrogen retention was approximately proportional to the decrement in inulin clearance. Only in G. B. (Table III) was there a decrease in the inulin clearance without alteration in blood urea 
nitrogen concentration. It seems unlikely that any defect in the inulin clearance as a measure of filtration under these conditions can account for a change of the magnitude observed (to 50, 58,60 , and 60 per cent of control-Table III) and these figures may be accepted, therefore, as evidence for diminished filtration. $\mathrm{PAH}$ clearance also decreased (Table III) and it may be presumed that intrarenal vasoconstriction lowered renal blood flow and filtration pressure, thus accounting for the fall in filtration rate. $\mathrm{PAH}$ clearance decreased by 27 per cent in the normal subject (A. C.-Table III) on the same regimen, without change in the inulin clearance. The renal extraction is always impaired in renal insufficiency (9) and it is possible that the decrement in $\mathrm{PAH}$ clearance was attributable, at least in part, to further reduction in extraction. Measurements of renal extraction of $\mathrm{PAH}$ before and after sodium deprivation are necessary to clarify this point.

The fall in filtration accounted not only for retention of nitrogen but it was also responsible for the fall in sodium excretion in patients with renal insufficiency. In the normal subject (A. C.
Table III) increased tubular reabsorption alone appeared to be sufficient. To test this point, a normal subject (A. B.) in whom electrolyte and water balance were carefully controlled was placed on a more rigorous regimen of dietary salt restriction (18.5 mEq. per day) and resin administration (120 gm. per day in divided doses) (Table V). Within a period of five days there was a net loss of $214 \mathrm{mEq}$. of potassium and $529 \mathrm{mEq}$. of sodium (or almost twenty times that observed in the uremic patient [T. C.] under the usual regimen). The glomerular filtration rate following this period was $120 \mathrm{ml}$. per min., a value well within normal limits, and there was no evidence of phosphate or nitrogen retention. These results agree with those of Wiggins and his associates (7) who found that the glomerular filtration rate did not change significantly and that nitrogen retention did not occur in 17 normal subjects receiving a low salt diet and $2 \mathrm{ml}$. of a mercurial diuretic (Mercuzanthin) daily, a regimen sufficient to produce significant reduction in plasma sodium concentration and in body weight during a three-day period.

Sodium output fell proportionately more than

TABLE IV

Sodium, potassium, and water balance during sodium deprivation and repletion in chronic uremia

\begin{tabular}{|c|c|c|c|c|c|c|c|c|c|c|c|c|}
\hline \multirow{2}{*}{$\begin{array}{l}\text { T. C. } \\
\text { Period }\end{array}$} & \multicolumn{2}{|c|}{ Uremia } & \multicolumn{4}{|c|}{$\begin{array}{l}\text { Sodium } \\
m E q .124 h r . \\
\text { Excretion }\end{array}$} & \multicolumn{4}{|c|}{$\begin{array}{l}\text { Potassium } \\
m E q . / 24 h r \\
\text { Excretion }\end{array}$} & \multicolumn{2}{|c|}{ Fluid } \\
\hline & $\begin{array}{l}\text { Hosp. } \\
\text { day }\end{array}$ & $\begin{array}{l}\text { Body } \\
\text { wt. }\end{array}$ & Intake & Urine & Stool & Balance & Intake & Urine & Stool & Balance & Intake & volume \\
\hline \multirow[t]{2}{*}{ Controlf } & $\begin{array}{l}4 \\
5\end{array}$ & $\begin{array}{l}K g . \\
61.6 \\
61.9\end{array}$ & $\begin{array}{l}63.0^{*} \\
23.6\end{array}$ & $\begin{array}{l}31.9 \\
11.6\end{array}$ & $\begin{array}{l}8.5 \\
8.5\end{array}$ & $\begin{array}{l}+22.6 \\
+\quad 3.5\end{array}$ & $\begin{array}{l}92.4 \\
92.4\end{array}$ & $\begin{array}{l}68.6 \\
72.6\end{array}$ & $\begin{array}{l}18.3 \\
18.3\end{array}$ & $\begin{array}{l}+5.5 \\
+1.5\end{array}$ & $\begin{array}{l}1,930 \\
1,930\end{array}$ & $\begin{array}{l}1,625 \\
1,680\end{array}$ \\
\hline & & & \multicolumn{4}{|c|}{$\begin{array}{c}\text { Net balance } \\
\text { Total exch. body } \mathrm{Na}=2,475 \mathrm{mEq} .\end{array}$} & \multicolumn{4}{|c|}{$\begin{array}{c}\text { Net balance } \\
\text { Total exch. body } \mathrm{K}=2,355 \mathrm{mEq} .\end{array}$} & & \\
\hline \multirow[t]{2}{*}{ Na depriv. } & $\begin{array}{r}6 \\
7 \\
8 \\
9 \\
10\end{array}$ & $\begin{array}{l}61.5 \\
61.5 \\
61.2 \\
61.1 \\
60.8\end{array}$ & $\begin{array}{l}23.6 \\
23.6 \\
23.6 \\
23.6 \\
23.6\end{array}$ & $\begin{array}{l}6.3 \\
7.4 \\
6.7 \\
4.5 \\
6.0\end{array}$ & $\begin{array}{l}23.2 \\
23.2 \\
23.2 \\
23.2 \\
23.2\end{array}$ & $\begin{array}{l}-\quad 5.8 \\
=\quad 7.0 \\
=\quad 6.3 \\
=\quad 4.1 \\
-\quad 5.6\end{array}$ & $\begin{array}{l}182.4 \dagger \\
182.4 \\
182.4 \\
182.4 \\
182.4\end{array}$ & $\begin{array}{l}76.4 \\
62.0 \\
80.0 \\
63.2 \\
59.6\end{array}$ & $\begin{array}{l}111.0 \\
111.0 \\
111.0 \\
111.0 \\
111.0\end{array}$ & $\begin{array}{r}-5.0 \\
+9.4 \\
-8.6 \\
+8.2 \\
+11.8\end{array}$ & $\begin{array}{l}1,930 \\
1,930 \\
1,930 \\
1,930 \\
1,930\end{array}$ & $\begin{array}{l}1,330 \\
1,405 \\
1,530 \\
1,200 \\
1,460\end{array}$ \\
\hline & & & \multicolumn{4}{|c|}{$\begin{array}{c}\text { Net balance } \\
\text { Total exch. body } \mathrm{Na}=2,440 \mathrm{mEq} .\end{array}$} & \multicolumn{4}{|c|}{$\begin{array}{cc}\text { Net balance } & +15.8 \\
\text { Total exch. body } \mathrm{K} & =2,410 \mathrm{mEq} .\end{array}$} & & \\
\hline \multirow[t]{2}{*}{$\underset{\text { Control }}{\text { III }}$} & $\begin{array}{l}11 \\
12\end{array}$ & $\begin{array}{l}60.5 \\
61.1\end{array}$ & $\begin{array}{l}61.9^{*} \\
20.5\end{array}$ & $\begin{array}{l}6.0 \\
7.4\end{array}$ & $\begin{array}{l}6.1 \\
6.1\end{array}$ & $\begin{array}{l}+49.8 \\
+\quad 7.0\end{array}$ & $\begin{array}{l}102.5 \\
102.5\end{array}$ & $\begin{array}{l}50.2 \\
41.5\end{array}$ & $\begin{array}{l}61.2 \\
61.2\end{array}$ & $\begin{array}{l}-8.9 \\
-0.2\end{array}$ & $\begin{array}{l}1,930 \\
1,930\end{array}$ & $\begin{array}{l}1,520 \\
1,460\end{array}$ \\
\hline & & & \multicolumn{4}{|c|}{ Net balance } & \multicolumn{3}{|c|}{ Net balance } & -9.1 & & \\
\hline \multirow[t]{2}{*}{$\stackrel{\text { IV }}{\text { Repletion }}$} & $\begin{array}{l}13 \\
14 \\
15 \\
16 \\
17\end{array}$ & $\begin{array}{l}60.7 \\
60.5 \\
60.9 \\
60.7 \\
60.9\end{array}$ & $\begin{array}{l}174.5 \\
174.5 \\
225.5 \\
225.5 \\
225.5\end{array}$ & $\begin{array}{r}13.5 \\
53.9 \\
140.8 \\
190.0 \\
212.2\end{array}$ & $\begin{array}{l}12.6 \\
12.6 \\
12.6 \\
12.6 \\
12.6\end{array}$ & $\begin{array}{r}+148.4 \\
+108.0 \\
+72.1 \\
+\quad 22.9 \\
+\quad 0.7\end{array}$ & $\begin{array}{l}102.5 \\
102.5 \\
102.5 \\
102.5 \\
102.5\end{array}$ & $\begin{array}{l}52.5 \\
69.2 \\
71.2 \\
71.4 \\
65.9\end{array}$ & $\begin{array}{l}16.1 \\
16.1 \\
16.1 \\
16.1 \\
16.1\end{array}$ & $\begin{array}{l}+34.2 \\
+17.2 \\
+15.2 \\
+15.0 \\
+20.5\end{array}$ & $\begin{array}{l}1,930 \\
1,930 \\
1,930 \\
1,930 \\
1,930\end{array}$ & $\begin{array}{l}1,410 \\
1,420 \\
1,675 \\
1,710 \\
1,710\end{array}$ \\
\hline & & & \multicolumn{4}{|c|}{$\begin{array}{l}\text { Net balance } \\
\text { ch. body } \mathrm{Na}=2,885 \mathrm{mEq}\end{array}$} & Total e & $\begin{array}{l}\text { bala } \\
\text { ch. boc }\end{array}$ & $\begin{array}{l}\text { ce } \\
\mathrm{K}=\end{array}$ & $\begin{array}{l}+102.1 \\
, 535 \mathrm{mEq}\end{array}$ & & \\
\hline
\end{tabular}

* Denotes additional sodium administered as sodium $p$-aminohippurate in measuring $\mathrm{Tm}_{\text {pah }}$.

$\dagger$ Denotes additional potassium administered in sodium removing resins (each $1 \mathrm{gm}$. resin contained $1 \mathrm{mEq}$. of potassium).

‡ Regimen in each period as noted under Table II. 
TABLE V

Sodium, potassium, and water balance during sodium depletion and repletion in normal man

\begin{tabular}{|c|c|c|c|c|c|c|c|c|c|c|c|c|}
\hline \multirow{2}{*}{$\begin{array}{l}\text { A. B. } \\
\text { Period }\end{array}$} & \multicolumn{2}{|c|}{ Normal } & \multicolumn{4}{|c|}{$\begin{array}{l}\text { Sodium } \\
m E q . / 24 \text { hr. } \\
\text { Excretion }\end{array}$} & \multirow{2}{*}{\multicolumn{4}{|c|}{$\begin{array}{l}\text { Potassium } \\
m E q . / 24 \mathrm{hr} . \\
\text { Excretion }\end{array}$}} & \multicolumn{2}{|c|}{$\underset{m l . / 24 h r}{\text { Fluid }}$} \\
\hline & $\underset{\text { day }}{\text { Hosp. }}$ & $\begin{array}{l}\text { Body } \\
\text { wt. }\end{array}$ & Intake & $\begin{array}{c}\text { Excr } \\
\text { Urine }\end{array}$ & etion & Balance & Intake & & & Balance & Intake & $\begin{array}{l}\text { Urine } \\
\text { volume }\end{array}$ \\
\hline \multirow{3}{*}{ Na deplet. $\ddagger$} & 10 & $\begin{array}{l}K g . \\
66.7\end{array}$ & \multicolumn{10}{|c|}{ Total exch. body $\mathrm{Na}=2,730 \mathrm{mEq}$. } \\
\hline & $\begin{array}{l}11 \\
12 \\
13 \\
14 \\
15\end{array}$ & $\begin{array}{l}66.1 \\
65.5 \\
65.2 \\
65.2 \\
64.7\end{array}$ & $\begin{array}{l}18.5 \\
18.5 \\
18.5 \\
18.5 \\
18.5\end{array}$ & $\begin{array}{l}0.4 \\
0.3 \\
0.4 \\
0.2 \\
0.5\end{array}$ & $\begin{array}{l}130.0 \\
130.0 \\
120.0 \\
120.0 \\
120.0\end{array}$ & $\begin{array}{l}-111.9 \\
-111.8 \\
-101.9 \\
-101.7 \\
-102.0\end{array}$ & $\begin{array}{l}257.3 \dagger \\
257.3 \\
257.3 \\
257.3 \\
257.3\end{array}$ & $\begin{array}{r}25.2 \\
18.8 \\
14.2 \\
9.2 \\
9.3\end{array}$ & $\begin{array}{l}290.0 \\
290.0 \\
281.4 \\
281.4 \\
281.4\end{array}$ & $\begin{array}{l}-57.9 \\
-51.5 \\
-38.3 \\
-33.3 \\
-33.4\end{array}$ & $\begin{array}{l}1,900 \\
1,900 \\
1,900 \\
1,900 \\
1,900\end{array}$ & $\begin{array}{l}1,810 \\
2,090 \\
1,970 \\
1,840 \\
1,730\end{array}$ \\
\hline & & & \multicolumn{4}{|c|}{$\begin{aligned} \text { Net balance } & =-529.3 \\
\text { Total exch. body } \mathrm{Na} & =2,132 \mathrm{mEq} .\end{aligned}$} & \multicolumn{3}{|c|}{ Net balance $=$} & -214.4 & & \\
\hline \multirow[t]{2}{*}{$\begin{array}{l}\text { II } \\
\text { Control }\end{array}$} & $\begin{array}{l}16 \\
17\end{array}$ & $\begin{array}{l}64.5 \\
64.3\end{array}$ & $\begin{array}{l}18.5 \\
61.3^{*}\end{array}$ & $\begin{array}{l}0.4 \\
0.5\end{array}$ & $\begin{array}{l}51.5 \\
51.5\end{array}$ & $\begin{array}{l}-33.4 \\
+\quad 9.3\end{array}$ & $\begin{array}{l}77.3 \\
77.3\end{array}$ & $\begin{array}{l}5.0 \\
3.9\end{array}$ & $\begin{array}{l}149.2 \\
149.2\end{array}$ & $\begin{array}{l}-76.9 \\
-75.8\end{array}$ & $\begin{array}{l}1,900 \\
1,900\end{array}$ & $\begin{array}{l}1,440 \\
1,860\end{array}$ \\
\hline & & & \multicolumn{3}{|c|}{ Net balance $=$} & -24.1 & \multicolumn{3}{|c|}{ Net balance $=$} & -152.7 & & \\
\hline \multirow[t]{2}{*}{$\begin{array}{c}\text { III } \\
\text { Repletion }\end{array}$} & $\begin{array}{l}18 \\
19 \\
20 \\
21 \\
22\end{array}$ & $\begin{array}{l}64.0 \\
64.6 \\
65.6 \\
65.8 \\
66.2\end{array}$ & $\begin{array}{l}103.5 \\
103.5 \\
103.5 \\
103.5 \\
103.5\end{array}$ & $\begin{array}{r}0.6 \\
0.8 \\
1.9 \\
10.9 \\
19.8\end{array}$ & $\begin{array}{l}1.5 \\
1.5 \\
1.5 \\
1.5 \\
1.5\end{array}$ & $\begin{array}{r}+101.4 \\
+101.2 \\
+100.1 \\
+91.1 \\
+\quad 82.2\end{array}$ & $\begin{array}{l}77.3 \\
77.3 \\
77.3 \\
77.3 \\
77.3\end{array}$ & $\begin{array}{r}3.1 \\
10.5 \\
29.8 \\
54.0 \\
50.0\end{array}$ & $\begin{array}{l}12.3 \\
12.3 \\
12.3 \\
12.3 \\
12.3\end{array}$ & $\begin{array}{r}+61.9 \\
+54.5 \\
+35.2 \\
+11.0 \\
+15.0\end{array}$ & $\begin{array}{l}1,900 \\
1,900 \\
1,900 \\
1,900 \\
1,900\end{array}$ & $\begin{array}{l}920 \\
900 \\
810 \\
900 \\
750\end{array}$ \\
\hline & & & \multicolumn{4}{|c|}{$\begin{aligned} \text { Net balance } & =+476.0 \\
\text { Total exch. body } \mathrm{Na} & =3,010 \mathrm{mEq} .\end{aligned}$} & \multicolumn{3}{|c|}{ Net balance $=$} & +177.6 & & \\
\hline
\end{tabular}

* Denotes additional sodium administered as sodium $p$-aminohippurate in measuring $\mathrm{Tm}_{\mathrm{pah}}$.

$\dagger$ Denotes additional potassium administered in sodium removing resins (each $1 \mathrm{gm}$. resin contained $1.5 \mathrm{mEq}$. of potassium).

$\ddagger \mathrm{Na}$ deplet.: sodium intake reduced to $18.5 \mathrm{mEq}$. per day and $120 \mathrm{gm}$. of a cation exchange resin (Carks-resin) given each day in divided doses. Regimen and abbreviations otherwise as under Table II.

filtration in G. B., J. F., and T. C. (Table III), in- deprivation. Tubular dysfunction was also apdicating that augmented tubular reabsorption also parent in a fall in $T \mathrm{~m}_{\mathrm{pah}}$ in 4 of the 5 uremics. may play a role in sodium conservation in uremia. There was no change in the lack of ability to In M. B. tubular reabsorption was greatly im- acidify the urine during PAH loading (Table II). paired and showed no evidence of increase during Cessation of resin administration and the addi-

TABLE VI

Effect of sodium deprivation on the volume of the body fluid compartments in patients with chronic renal insufficiency

\begin{tabular}{|c|c|c|c|c|c|c|c|c|c|c|c|c|c|}
\hline \multirow{2}{*}{$\begin{array}{l}\text { Patient } \\
\text { G. B. }\end{array}$} & \multirow{2}{*}{\begin{tabular}{l}
\multicolumn{1}{c}{ Regimen* } \\
Control \\
Na depriv.
\end{tabular}} & \multirow{2}{*}{$\begin{array}{c}\begin{array}{c}\text { Body } \\
\text { wt. }\end{array} \\
\\
K_{g} . \\
62.6 \\
62.5\end{array}$} & \multicolumn{2}{|c|}{$\begin{array}{c}\text { Total body } \\
\text { water }\end{array}$} & \multicolumn{2}{|c|}{$\begin{array}{l}\text { Extracellular } \\
\text { fluid }\end{array}$} & \multicolumn{2}{|c|}{$\begin{array}{l}\text { Intracellular } \\
\text { water }\end{array}$} & \multicolumn{2}{|c|}{$\begin{array}{l}\text { Plasma } \\
\text { volume }\end{array}$} & \multicolumn{2}{|c|}{$\begin{array}{l}\text { Blood } \\
\text { volume }\end{array}$} & \multirow[b]{2}{*}{$\begin{array}{c}\text { Het } \\
\\
\% \\
38 \\
31\end{array}$} \\
\hline & & & $\begin{array}{c}L . \\
31.0 \\
30.0\end{array}$ & 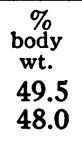 & $\begin{array}{c}L . \\
14.5 \\
16.5\end{array}$ & $\begin{array}{c}\begin{array}{c}\% \\
\text { body } \\
\text { wt. }\end{array} \\
23.2 \\
26.5\end{array}$ & $\begin{array}{c}L . \\
16.5 \\
13.5\end{array}$ & $\begin{array}{c}\begin{array}{c}\% \\
\text { body } \\
\text { wt. }\end{array} \\
26.5 \\
21.6\end{array}$ & $\begin{array}{c}L . \\
3.9 \\
4.3\end{array}$ & $\begin{array}{c}\% \\
\text { body } \\
\text { wt. } \\
6.3 \\
6.9\end{array}$ & $\begin{array}{c}L . \\
6.4 \\
6.3\end{array}$ & $\begin{array}{c}\begin{array}{c}\% \\
\text { body } \\
\text { wt. }\end{array} \\
10.0 \\
10.0\end{array}$ & \\
\hline M. B. & $\begin{array}{l}\text { Control } \\
\mathrm{Na} \text { depriv. }\end{array}$ & $\begin{array}{l}68.5 \\
67.5\end{array}$ & $\begin{array}{l}37.5 \\
32.5\end{array}$ & $\begin{array}{l}54.8 \\
48.0\end{array}$ & $\begin{array}{l}14.5 \\
15.5\end{array}$ & $\begin{array}{l}21.2 \\
23.0\end{array}$ & $\begin{array}{l}23.0 \\
17.0\end{array}$ & $\begin{array}{l}33.5 \\
25.2\end{array}$ & $\begin{array}{l}3.6 \\
3.8\end{array}$ & $\begin{array}{l}5.2 \\
5.6\end{array}$ & $\begin{array}{l}5.0 \\
5.1\end{array}$ & $\begin{array}{l}7.3 \\
.7 .5\end{array}$ & $\begin{array}{l}32 \\
25\end{array}$ \\
\hline C. P. & $\begin{array}{l}\text { Control } \\
\text { Na depriv. } \\
\text { Repletion }\end{array}$ & $\begin{array}{l}82.0 \\
81.2 \\
84.0\end{array}$ & $\begin{array}{l}41.0 \\
44.0 \\
45.5\end{array}$ & $\begin{array}{l}50.0 \\
54.0 \\
54.2\end{array}$ & $\begin{array}{l}16.5 \\
13.0 \\
16.5\end{array}$ & $\begin{array}{l}20.2 \\
16.0 \\
19.6\end{array}$ & $\begin{array}{l}24.5 \\
31.0 \\
29.0\end{array}$ & $\begin{array}{l}30.0 \\
38.2 \\
34.5\end{array}$ & $\begin{array}{l}3.7 \\
3.9 \\
3.7\end{array}$ & $\begin{array}{l}4.6 \\
4.8 \\
4.4\end{array}$ & $\begin{array}{l}5.5 \\
5.7 \\
5.8\end{array}$ & $\begin{array}{l}6.7 \\
7.0 \\
6.9\end{array}$ & $\begin{array}{l}33 \\
32 \\
29\end{array}$ \\
\hline J. F. & $\begin{array}{l}\text { Control } \\
\text { Na depriv. } \\
\text { Repletion }\end{array}$ & $\begin{array}{l}50.2 \\
48.6 \\
51.4\end{array}$ & $\begin{array}{l}31.0 \\
30.5 \\
31.0\end{array}$ & $\begin{array}{l}61.2 \\
62.8 \\
60.2\end{array}$ & $\begin{array}{l}12.5 \\
12.0 \\
15.0\end{array}$ & $\begin{array}{l}25.0 \\
24.6 \\
29.0\end{array}$ & $\begin{array}{l}18.5 \\
18.5 \\
16.0\end{array}$ & $\begin{array}{l}36.2 \\
39.0 \\
31.2\end{array}$ & $\begin{array}{l}2.9 \\
3.0 \\
3.3\end{array}$ & $\begin{array}{l}5.9 \\
6.2 \\
6.3\end{array}$ & $\begin{array}{l}4.2 \\
4.2 \\
4.3\end{array}$ & $\begin{array}{l}8.3 \\
8.7 \\
8.4\end{array}$ & $\begin{array}{l}29 \\
29 \\
22\end{array}$ \\
\hline T. C. & $\begin{array}{l}\text { Control } \\
\text { Na depriv. } \\
\text { Repletion }\end{array}$ & $\begin{array}{l}61.6 \\
60.8 \\
60.9\end{array}$ & $\begin{array}{l}31.0 \\
30.0 \\
31.0\end{array}$ & $\begin{array}{l}50.2 \\
49.5 \\
51.0\end{array}$ & $\begin{array}{l}15.5 \\
15.0 \\
16.0\end{array}$ & $\begin{array}{l}25.2 \\
24.6 \\
26.4\end{array}$ & $\begin{array}{l}15.5 \\
15.0 \\
15.0\end{array}$ & $\begin{array}{l}25.2 \\
24.6 \\
24.6\end{array}$ & $\begin{array}{l}2.9 \\
3.1 \\
3.4\end{array}$ & $\begin{array}{l}4.7 \\
5.1 \\
5.5\end{array}$ & $\begin{array}{l}4.9 \\
4.9 \\
4.9\end{array}$ & $\begin{array}{l}8.1 \\
8.1 \\
8.1\end{array}$ & $\begin{array}{l}43 \\
36 \\
32\end{array}$ \\
\hline
\end{tabular}

* Regimen as noted under Table II. 
TABLE VII

Comparison of simultaneous measurements of the four-hour $\mathrm{Na}^{21}$ space and the inulin space in patients with chronic renal insufficiency

\begin{tabular}{|c|c|c|c|c|c|c|}
\hline Patient & Regimen* & $\begin{array}{c}\text { Body } \\
\text { wt. } \\
\text { kg. }\end{array}$ & $\begin{array}{l}\mathrm{Na}^{24} \\
L .\end{array}$ & $\begin{array}{c}\text { space } \\
\% \\
\text { body } \\
\text { wt. }\end{array}$ & Inulin & $\begin{array}{c}\text { space } \\
\% \\
\text { body } \\
\text { wt. }\end{array}$ \\
\hline $\begin{array}{l}\text { C. P. } \\
\text { T. C. } \\
\text { I. F. }\end{array}$ & $\begin{array}{l}\text { Control } \\
\text { Control } \\
\text { Na depriv. } \\
\text { Repletion } \\
\text { Repletion }\end{array}$ & $\begin{array}{l}82.0 \\
61.6 \\
60.8 \\
60.9 \\
51.4\end{array}$ & $\begin{array}{l}22.0 \\
18.8 \\
18.0 \\
19.5 \\
18.4\end{array}$ & $\begin{array}{l}27.0 \\
30.5 \\
29.6 \\
32.0 \\
35.8\end{array}$ & $\begin{array}{l}16.5 \\
15.5 \\
14.9 \\
16.1 \\
14.9\end{array}$ & $\begin{array}{l}20.0 \\
25.2 \\
24.5 \\
26.4 \\
29.0\end{array}$ \\
\hline
\end{tabular}

* Regimen as noted under Table II.

tion of salt to the diet resulted in a tendency for all values (except $\mathrm{Tm}_{\mathrm{pah}}$ ) to return to or rise above the control levels in 3 uremic patients (C. P., J. F., and T. C.-Repletion, Table III), indicating the reversibility of the changes occurring during deprivation. The failure of $\mathrm{Tm}_{\mathrm{pah}}$ to recover has also been noted by Chasis and his co-workers (29) in a study of the effect of salt restriction in patients with essential hypertension.

Body water and electrolyte distribution during renal insufficiency. Measurements of the volume of total body water (antipyrine "space") in five patients with renal insufficiency (Table VI) yielded values well within normal limits, ranging from 49.5 to 61.2 per cent of body weight. Since all were spare and non-edematous this finding was not unexpected. But the inulin "space" (extracellular water volume) was greater than normal in every instance, ranging from 20.2 to 25.2 per cent (in contrast to the normal range from 11.4 to 18.0 per cent of body weight [30]) and the volume of intracellular fluid was correspondingly reduced. Determination of the volume of distribution of radiosodium four hours after administration in three of these subjects also yielded higher than normal values (Table VII). As in normals, the four-hour radiosodium "space" (21) exceeded the inulin "space." The blood volumes ( $T-1824)$ were all within normal limits.

The "total exchangeable" sodium and potassium were within normal limits in three subjects, two of whom were studied during the control period (C. P. and T. C.-Table VIII) and one during sodium repletion only ( $\mathrm{J}$. F.-Table VIII). Calculation of the electrolyte concentrations within the "cells" (and bone), in these patients (using simultaneously determined antipyrine and inulin "spaces") revealed concentrations of 26,22 , and $28 \mathrm{mEq}$. sodium per liter of cell fluid (normal range -31 to $44 \mathrm{mEq}$. per liter [31]) and 105, 150 , and $120 \mathrm{mEq}$. potassium per liter (normal range -96 to $125 \mathrm{mEq}$. per liter [31]) suggesting that loss of sodium from the cells and/or bone may have occurred during the course of renal insufficiency despite maintenance of extracellular fluid concentrations. However, the validity of the methods available for the estimation of extracellular fluid volume is open to serious question in uremia where changes in cellular permeability could permit rapid escape of inulin or radioactive sodium into the cells and this conclusion cannot be accepted without reserve.

Effect of sodium deprivation on body water and electrolyte distribution. Deprivation of sodium did not alter the volume or distribution of body water

TABLE VIII

The distribution of sodium and potassium in patients with chronic renal insufficiency

\begin{tabular}{|c|c|c|c|c|c|c|c|c|c|c|c|c|c|c|c|}
\hline Patient & Regimen* & $\begin{array}{l}\text { Body } \\
\text { wt. }\end{array}$ & $\begin{array}{l}\text { Total } \\
\text { body } \\
\text { water }\end{array}$ & $\begin{array}{l}\text { Extra- } \\
\text { cell. } \\
\text { fluid }\end{array}$ & $\begin{array}{l}\text { Intra- } \\
\text { cell.t } \\
\text { fluid }\end{array}$ & $\underset{\mathrm{Na}}{\text { Plasma }}$ & $\begin{array}{l}\text { Total } \\
\text { Na }\end{array}$ & $\begin{array}{l}\text { Extra- } \\
\text { cell. } \\
\mathrm{Na}\end{array}$ & $\begin{array}{l}\text { Intra- } \\
\text { cell. } \\
\mathrm{Na}\end{array}$ & $\begin{array}{l}\text { Intra- } \\
\text { cell. } \\
\text { Na } \\
\text { conc. }\end{array}$ & $\underset{\mathbf{K}}{\text { Plasma }}$ & $\underset{\mathbf{K}}{\text { Total }}$ & $\begin{array}{l}\text { Extra- } \\
\text { cell. } \\
\mathbf{K}\end{array}$ & $\begin{array}{l}\text { Intra- } \\
\text { cell. } \\
\mathbf{K}\end{array}$ & $\begin{array}{l}\text { Intra- } \\
\text { cell. } \\
\mathbf{K} \\
\text { conc. }\end{array}$ \\
\hline C. $\mathbf{P}$. & Control & $\begin{array}{l}K g . \\
82.0\end{array}$ & 40.9 & $\begin{array}{r}\text { Liters } \\
16.5\end{array}$ & 24.4 & $\begin{array}{c}m E q . / L \\
135\end{array}$ & 2,870 & $\begin{array}{c}m E q . \\
2,227\end{array}$ & 643 & $\begin{array}{c}\text { mEq.I } \\
\text { "cell" } \\
\text { woater } \\
26\end{array}$ & $\begin{array}{l}\text { mEq.l } \\
\text { L. } \\
3.8\end{array}$ & 2,633 & $\begin{array}{c}m E q . \\
63\end{array}$ & 2,570 & $\begin{array}{c}\text { mEq./ } \\
L \text { cell" } \\
\text { water } \\
105\end{array}$ \\
\hline T. C. & $\begin{array}{l}\text { Control } \\
\text { Na depriv. } \\
\text { Repletion }\end{array}$ & $\begin{array}{l}61.6 \\
60.8 \\
60.9\end{array}$ & $\begin{array}{l}30.7 \\
30.1 \\
31.1\end{array}$ & $\begin{array}{l}15.5 \\
14.9 \\
16.1\end{array}$ & $\begin{array}{l}15.2 \\
15.2 \\
15.0\end{array}$ & $\begin{array}{l}138 \\
137 \\
140\end{array}$ & $\begin{array}{l}2,475 \\
2,440 \\
2,885\end{array}$ & $\begin{array}{l}2,140 \\
2,040 \\
2,253\end{array}$ & $\begin{array}{l}335 \\
400 \\
632\end{array}$ & $\begin{array}{l}22 \\
26 \\
42\end{array}$ & $\begin{array}{l}5.0 \\
4.1 \\
4.7\end{array}$ & $\begin{array}{l}2,355 \\
2,410 \\
2,535\end{array}$ & $\begin{array}{l}78 \\
61 \\
76\end{array}$ & $\begin{array}{l}2,277 \\
2,349 \\
2,459\end{array}$ & $\begin{array}{l}150 \\
155 \\
164\end{array}$ \\
\hline J. F. & Repletion & 51.4 & 30.7 & 14.9 & 15.8 & 136 & 2,478 & 2,028 & 450 & 28 & 5.0 & 1,962 & 75 & 1,887 & 120 \\
\hline
\end{tabular}

* Regimen as noted under Table II.

† The term "intracellular" as used here must include a portion of the fluid and electrolytes within the bones since it is probable that inulin does not readily diffuse into this "space" during the time allotted for equilibration. 
in any consistent manner. Body weight did not change or it decreased by no more than $1.6 \mathrm{Kg}$. (J. F.-Table III). Total body water (Table VI) remained more or less unchanged in two (J. F. and T. C.), rose in one (C. P.), and fell in two (G. B. and M. B.). Likewise the inulin "space" rose slightly in two (M. B. and G. B.) and fell somewhat in the remainder. In T. C. (Table VII) the four-hour radiosodium "space" and inulin "space" behaved similarly in showing a slight diminution of the same magnitude. The blood volume remained unaltered although the hematocrit fell in three subjects. (It might be added here that arterial pressures and pulse rates did not change and all patients remained well and capable of adjusting normally to positional shifts.)

The "total exchangeable" sodium and potassium in T. C. (Table VIII) were not affected significantly by sodium deprivation although the balance study (Table IV) revealed a net loss of $28.8 \mathrm{mEq}$. of sodium in close agreement with the decrement of $35 \mathrm{mEq}$. in "total exchangeable" sodium.

Despite the uncertainties of the methods employed it seems probable that the agreement between values obtained before and after sodium deprivation indicates relatively little real change in body water volume or distribution during deprivation. The fact that sodium repletion resulted in sizeable increments in the inulin "space" in C. P., J. F., and T. C. strengthens the view that this method is capable of indicating at least the relative magnitude and direction of change in extracellular fluid volume. And, since calculation of intracellular (and bone) electrolyte concentration depends on this figure, sodium concentration within the "cells" probably changed very little during sodium deprivation.

\section{DISCUSSION}

Sodium deprivation without depletion produces effects in patients with chronic renal insufficiency that differ qualitatively as well as quantitatively from those observed in normal persons. In four of five uremic patients the glomerular filtration rate decreased markedly in association with a fall in effective renal plasma flow during a five day period of deprivation. In all but one the output of sodium diminished relatively more than filtration indicating that increased tubular reab- sorption of sodium also played a part in the response. The reduction in filtration was attended by a slight increase in serum inorganic phosphate concentration in all and by elevations in blood urea nitrogen concentration in three. In a normal individual under the same conditions urinary sodium output was far more drastically curtailed by increased tubular reabsorption alone. Even when normal persons are subjected to sodium depletion as in one subject of this study and in 17 studies by Wiggins and his associates (7), tubular reabsorption increases with little or no change in filtration. In large part this difference in response is traceable to the underlying abnormality in renal structure and function.

Uremia in chronic disorders of the kidney develops primarily on the basis of impaired filtration (9). Derangements in renal hemodynamics as well as destruction of tubules and glomeruli contribute to this defect. Hypofiltration is associated with what appears at first sight to be an even more serious disorder of tubular function. Reabsorption is greatly reduced permitting the escape of a much larger percentage of the filtered water and electrolytes than in the normal. Indeed, as in five of the eight patients examined in this study (Table I) the output of potassium may be several times in excess of the filtered load, indicating tubular secretion. Osmotic nephron diuresis as a result of the elevation in the plasma concentration of urea and other osmotically active substances may play a role in causing water and electrolyte loss. Mudge, Foulks, and Gilman (32) have shown that as much as 50 per cent of the filtered water $\left(\mathrm{V} / \mathrm{C}_{\mathrm{in}}\right)$ may be excreted in the urine in dogs receiving large amounts of urea by infusion. In only one of the subjects in this series (Table I) $\operatorname{did} \mathrm{V} / \mathrm{C}_{\mathrm{in}}$ exceed 50 per cent and here the figure may be erroneuosly high owing to the low value for inulin clearance. Thus, the factor of osmotic diuresis seems a more likely cause for high values of $\mathrm{V} / \mathrm{C}_{\mathrm{in}}$ than the secretion of water by the tubules which Platt (33) has postulated.

This change in tubular activity is not to be viewed solely as the consequence of tubular destruction and glomerulo-tubular imbalance. Plasma electrolyte composition may be maintained within normal limits for years, as in some of the patients of this series (Table III) and it must be postulated that tubular and glomerular function 
are approximately integrated in some manner to serve the needs of chemical regulation. Thus the alterations in tubular function appear to be related to alterations in glomerular filtration; the data in Tables I to III suggest that reabsorption diminishes roughly in proportion to filtration with advance in the disease process and a fall in glomerular filtration. (This phenomenon contrasts strikingly with the tendency observed in normal individuals for reabsorption to increase relative to filtration when the filtration rate is lowered by one means or another [22]).

However, excesses or loss of water and electrolytes or the development of conditions such as congestive heart failure, hemorrhage, or vomiting unmasks or intensifies a fundamental defect in adaptation. Fixation of urinary specific gravity and reaction is a commonplace in renal insufficiency. The failure of the patients in this series to adjust to the obligatory excretion of an anion (PAH) by increasing the output of buffer (phosphate) and by lowering the urinary $\mathrm{pH}$ illustrates the inability of the nephrons to compensate adequately for acute change by altered tubular cellular activity. In this situation readjustments in tubular function seem to be impossible or greatly delayed and the only available corrective mechanism appears to be hemodynamic in character. Intrarenal vasoconstriction and reduction in glomerular filtration may be part of a widespread systemic circulatory response, as in shock or cardiac insufficiency, but, on the basis of the present study, it appears that such a generalized circulatory derangement detectable by present methods is not always necessary for renal vascular readjustments.

It is certainly true that disturbance in the volume and composition of body water are the rule in renal insufficiency. Although carefully selected, all five of the uremic subjects studied presented evidence of expansion of the volume of inulin and four-hour radiosodium distribution, which, with due allowance for the uncertainties of these methods, seems to indicate enlargement of the extracellular fluid volume and diminution of the intracellular (and bone) fluid. A similar change has been observed following starvation (34) where it is apparently attributable to partial replacement of lost tissue (accounting for the shrinkage in intracellular water volume) by additional interstitial fluid. Weight loss occurs commonly during renal insufficiency and it was prominent among the patients of this series. The reduction observed in intracellular (and bone) sodium concentration is not so easily explained. Since plasma sodium concentration was within normal limits in all, the sodium content of the extracellular fluid was apparently maintained at the expense of the "cells" and/or bone. This shift may have played a role in conditioning the renal functional response to sodium deprivation. Unfortunately the values yielded by the methods available to us are not only of uncertain validity but also of uncertain application. In the present case, the magnitude and uniform direction of the change observed provide reasons for accepting its validity. But information regarding the tissues chiefly affected by such a shift is of the first importance in assessing its effect on kidney function. This information is not adducible from these data because the value for intracellular sodium content applies to all the tissues and to none in particular. In any event, renal insufficiency seems capable of producing a disturbance in cellular electrolyte metabolism that is not immediately evident in an altered plasma electrolyte pattern.

Azotemia may occur following salt depletion in the absence of renal disease $(1,35)$ but some disturbance in cardiovascular function is nearly always detectable. Furthermore, marked changes in plasma electrolyte pattern usually develop. Both of these factors may operate to stimulate the kidney directly either by changing the pressure under which it is perfused, by exciting generalized compensatory cardiovascular adjustments in which the renal vasculature participates, or by direct action of altered plasma composition upon the nephrons. But none of these could be clearly defined as effective agents in causing the changes noted in the uremic subjects of this series during sodium deprivation. The arterial pressure and the blood volume remained constant throughout. No evidence of any change in the volume or distribution of body water was detectable and there was no remarkable change in plasma composition apart from the elevation in blood urea nitrogen and inorganic phosphate concentrations. Nevertheless, the plasma sodium concentration did decrease slightly in all and apparently there may have been a very small decrement in total sodium mass, to judge by one balance study and determi- 
nations of "total exchangeable" sodium in one individual. It is possibly this change, as small as it is, that sets in motion a reversible intrarenal vasoconstriction and subsequent diminution in glomerular filtration that gives rise to L'azotémie par manque de sel.

Evidently the same factors operate in the abnormal kidney as in the normal. Under the circumstances of sodium deprivation tubular reabsorption increases but to such a limited extent that dependence on this alone would not provide an adequate response to the need for sodium conservation. In the normal the response of the tubules is entirely adequate but the question may properly be raised whether a change in filtration of some kind does not also occur. This question has been much debated $(22,36)$ but lacks as yet any conclusive answer because it may be argued that the change required to be effective is too small for detection by the best methods now available. The increased importance of such a mechanism in the uremic patient may speak for its activity in the normal.

\section{SUMMARY}

Diminished urinary sodium excretion follows sodium deprivation without depletion by low sodium intake and small doses of a cation exchange resin (60 gm. per day). In five patients with chronic renal insufficiency secondary to chronic renal disease this response was attributable chiefly to diminished glomerular filtration (inulin clearance) apparently resulting from intrarenal vasoconstriction (a fall in $\mathrm{PAH}$ clearance) whereas increased tubular reabsorption alone was responsible in one normal subject.

In eight uremic patients, the fractions of filtered sodium, chloride, and water excreted in the urine under resting conditions were found to exceed the maximal values observed in normal men during imposition of loads of these substances. This "nephron diuresis" was associated, in five of the eight, with the excretion of a larger amount of potassium than could be accounted for by filtration alone, indicating tubular secretion of potassium. A study of electrolyte excretion during $\mathrm{PAH}$ loading in five revealed a fixation of urinary $\mathrm{pH}$ and inability to meet the need for obligatory excretion of an anion (PAH) by increased output of phosphate as a buffer, illustrating the lack of tubular responsivity to need.

Five uremic subjects in whom the volumes of distribution of antipyrine, inulin and T-1824 were determined presented evidence of an expansion of extracellular water (i.e., the volumes of distribution of both radiosodium four hours after administration and inulin were well above normal in all) at the expense of intracellular fluid volume, possibly as a result of inanition and loss of tissue. Using values for "total exchangeable" sodium and potassium obtained in three of these, calculation of intracellular sodium content yielded values well below the normal range.

Although these findings provide an explanation for the need to conserve sodium and for the particular renal mechanism available for the purpose, they shed no light on the manner in which the renal reaction was set in motion. No change in blood pressure, blood volume or the volumes and distribution of body fluids was evident. The blood urea nitrogen content rose in three individuals, the serum phosphate concentration increased in all, and serum sodium concentration decreased slightly. According to measurements of sodium balance and "total exchangeable" sodium in one patient, 28.8 to $35 \mathrm{mEq}$. of sodium were lost during the five day period of deprivation.

\section{ACKNOWLEDGMENTS}

We wish to express our deep appreciation to Mrs. Joan Banfield, Mrs. Lottie Klayman, Mrs. Michaeleen Patterson, Miss Emily Bidwell, Miss Margaret N. Hawthorne (head nurse, Metabolism Service), Miss Iva Moen, and Miss Mary Iles (dietitians, Metabolism Service) for their skillful and cheerful technical and professional assistance.

\section{REFERENCES}

1. Blum, L., Graber, P., and Caulaert, V., L'azotémie par manque de sel; dans le diabète grave. Ann. d. Méd., 1929, 25, 23.

2. Blum, L., Grabar, P., and Caulaert, V., L'azotémie par manque de sel; son méchanisme. Ann. d. Méd., 1929, 25, 34.

3. Peters, J. P., and Van Slyke, D. D., Quantitative Clinical Chemistry. Vol. I. Interpretations. Williams and Wilkins Company, Baltimore, 1931, pp. 1264.

4. McCance, R. A., Experimental sodium chloride deficiency in man. Proc. Roy. Soc., London, Series B, 1936, 119, 245. 
5. Elkinton, J. R., Danowski, T. S., and Winkler, A. W., Hemodynamic changes in salt depletion and in dehydration. J. Clin. Invest., 1946, 25, 120.

6. Landis, E. M., Elsom, K. A., Bott, P. A., and Shiels, E., Observations on sodium chloride restriction and urea clearance in renal insufficiency. J. Clin. Invest., $1935,14,525$.

7. Wiggins, W. S., Manry, C. H., Lyons, R. H., and Pitts, R. F., The effect of salt loading and salt depletion on rental function and electrolyte excretion in man. Circulation, 1951, 3, 275.

8. Goldring, W., and Chasis, H., Hypertension and $\mathrm{Hy}-$ pertensive Disease. The Commonwealth Fund, New York, 1944.

9. Bradley, S. E., Bradley, G. P., Tyson, C. J., Curry, J. J., and Blake, W. D., Renal function in renal diseases. Am. J. Med., 1950, 9, 766.

10. Van Slyke, D. D., and Neill, J. M., The determination of gases in blood and other solutions by vacuum extraction and manometric measurement. I. J. Biol. Chem., 1924, 61, 523.

11. Clarke, E. P., and Collip, J. B., A study of the Tisdall method for the determination of blood serum calcium with a suggested modification. J. Biol. Chem., 1925, 63, 461.

12. Gentzkow, C. J., An accurate method for the determination of blood urea nitrogen by direct Nesslerization. J. Biol. Chem., 1942, 143, 531.

13. Fox, C. L., Jr., Stable internal standard flame photometer for potassium and sodium analyses. Analyt. Chem., 1951, 23, 137.

14. Schales, O., and Schales, S. S., A simple and accurate method for the determination of chloride in biological fluids. J. Biol. Chem., 1941, 140, 879.

15. Fiske, C. H., and Subbarow, Y., The colorimetric determination of phosphorus. J. Biol. Chem., 1925, 66, 375 .

16. Soberman, R., Brodie, B. B., Levy, B. B., Axelrod, J., Hollander, V., and Steele, J. M., The use of antipyrine in the measurement of total body water in man. J. Biol. Chem. 1949, 179, 31.

17. Gaudino, M., Schwartz, I. L., and Levitt, M. F., Inulin volume of distribution as a measure of extracellular fluid in dog and man. Proc. Soc. Exper. Biol. \& Med., 1948, 68, 507.

18. Gregersen, M. I., A practical method for the determination of blood volume with the dye T-1824. A survey of the present basis of the dye-method and its clinical application. J. Lab. \& Clin. Med., 1944, 29, 1266.

19. Forbes, G. B., and Perley, A., Estimation of total body sodium by isotopic dilution. I. Studies on young adults. J. Clin. Invest., 1951, 30, 558.

20. Corsa, L., Jr., Olney, J. M., Jr., Steenburg, R. W., Ball, M. R., and Moore, F. D., The measurement of exchangeable potassium in man by isotope dilution. J. Clin. Invest., 1950, 29, 1280.

21. Kaltreider, N. L., Meneely, G. R., Allen, J. R., and Bale, W. F., Determination of the volume of the extracellular fluid of the body with radioactive sodium. J. Exper. Med., 1941, 74, 569.

22. Smith, H. W., The Kidney; Structure and Function in Health and Disease. Oxford University Press, New York, 1951.

23. Earle, D. P., Farber, S. J., Alexander, J. D., and Pellegrino, E. D., Renal function and electrolyte metabolism in acute glomerulonephritis. J. Clin. Invest., 1951, 30, 421.

24. Keith, N. M., King, H. E., and Osterberg, A. E., Serum concentration and renal clearance of potassium in severe renal insufficiency in man. Arch. Int. Meed., 1943, 71, 675.

25. Earle, D. P., Sherry, S., Eichna, L. W., and Conan, N. J., Low potassium syndrome due to defective renal tubular mechanisms for handling potassium. Am. J. Med., 1951, 11, 283.

26. Platt, R., Sodium and potassium excretion in chronic renal failure. Clin. Sc., 1950, 9, 367.

27. West, C. D., and Rapoport, S., Urinary excretion of phosphate following the injection of sodium $p$-aminohippurate. Proc. Soc. Exper. Biol. \& Med., 1949, 71, 322.

28. Stanbury, S. W., and Thomson, A. E., Diurnal variations in electrolyte excretion. Clin. Sc., 1951, 10, 267.

29. Chasis, H., Goldring, W., Breed, E. S., Schreiner, G. E., and Bolomey, A. A., Salt and protein restriction; effects on blood pressure and renal hemodynamics in hypertensive patients. J. A. M. A., 1950, 142, 711.

30. Schwartz, I. L., Schachter, D., and Freinkel, N., The measurement of extracellular fluid in man by means of a constant infusion technique. J. Clin. Invest., 1949, 28, 1117.

31. Deane, N., and Smith, H. W., The distribution of sodium and potassium in man. J. Clin. Invest., 1952, 31, 197.

32. Mudge, G. H., Foulks, J., and Gilman, A., Effect of urea diuresis on renal excretion of electrolytes. Am. J. Physiol., 1949, 158, 218.

33. Platt, R., Structural and functional adaptation in renal failure. Brit. M. J., 1952, 1, 1313 and 1372.

34. Henschel, A., Mickelsen, O., Taylor, H. L., and Keys, A., Plasma volume and thiocyanate space in famine edema and recovery. Am. J. Physiol., 1947, 150, 170.

35. Schroeder, H. A., Renal failure associated with low extracellular sodium chloride; the low salt syndrome. J. A. M. A., 1949, 141, 117.

36. Bradley, S. E., The Pathologic Physiology of Uremia in Chronic Bright's disease. American Lecture Series, No. 15, American Lectures in Physiology, Springfield, Ill., Charles C. Thomas, 1948.

37. Nickel, J. F., Lowrance, P., and Leifer, E., Effect of sodium depletion and repletion on renal function and body fluids during uremia. J. Clin. Invest., 1951, 30, 664. 